\title{
Electron Capture and Transfer Dissociation: Peptide Structure Analysis at Different Ion Internal Energy Levels
}

\author{
Hisham Ben Hamidane, ${ }^{\mathrm{a}}$ Diego Chiappe, ${ }^{\mathrm{b}}$ Ralf Hartmer, ${ }^{\mathrm{c}}$ \\ Aleksey Vorobyev, ${ }^{\mathrm{a}}$ Marc Moniatte, ${ }^{\mathrm{b}}$ and Yury O. Tsybin ${ }^{\mathrm{a}}$ \\ a Biomolecular Mass Spectrometry Laboratory, Ecole Polytechnique Fédérale de Lausanne, Lausanne, Switzerland \\ b Proteomics Core Facility, Ecole Polytechnique Fédérale de Lausanne, Lausanne, Switzerland \\ ${ }^{\mathrm{c}}$ Bruker Daltonics GmbH, Bremen, Germany
}

We decoupled electron-transfer dissociation (ETD) and collision-induced dissociation of charge-reduced species (CRCID) events to probe the lifetimes of intermediate radical species in ETD-based ion trap tandem mass spectrometry of peptides. Short-lived intermediates formed upon electron transfer require less energy for product ion formation and appear in regular ETD mass spectra, whereas long-lived intermediates require additional vibrational energy and yield product ions as a function of CRCID amplitude. The observed dependencies complement the results obtained by double-resonance electron-capture dissociation (ECD) Fourier transform ion cyclotron resonance mass spectrometry (FT-ICR MS) and ECD in a cryogenic ICR trap. Compared with ECD FT-ICR MS, ion trap MS offers lower precursor ion internal energy conditions, leading to more abundant charge-reduced radical intermediates and larger variation of product ion abundance as a function of vibrational post-activation amplitude. In many cases decoupled CRCID after ETD exhibits abundant radical $c$-type and even-electron $z$-type ions, in striking contrast to predominantly even-electron $c$-type and radical z-type ions in ECD FT-ICR MS and especially activated ion-ECD, thus providing a new insight into the fundamentals of ECD/ETD. (J Am Soc Mass Spectrom 2009, 20, 567-575) (C) 2009 Published by Elsevier Inc. on behalf of American Society for Mass Spectrometry

$\mathrm{D}$ evelopment of recent analytical methods for improved peptide and protein structural analysis has been directed by a combination of complementary tandem mass spectrometry (MS/MS) methods [1-3]. Particular advances have been achieved as a result of Fourier transform ion cyclotron resonance mass spectrometry (FT-ICR MS)-based electron capture dissociation (ECD) [4] complementarity to slow heating fragmentation methods [5], such as collision-induced dissociation (CID) [6] and infrared multiphoton dissociation (IRMPD) [7]. In addition to mainly product ion mass-based MS/ MS, product ion abundance (PIA) in ECD is increasingly considered as a new source of information to improve peptide and protein sequencing $[8,9]$, quantitative modification analysis [10, 11], higher-order structure characterization $[8,12-15]$, providing new insights into ECD mechanism [16, 17], suggesting charge location in peptides and proteins $[18,19]$, and indicating routes toward developing a quantitative model of ECD/ETD [15].

Double-resonance (DR) ECD, with and without ion preactivation, is used to estimate the radical intermediate lifetimes and differentiate between short-lived and long-lived intermediates by monitoring PIA variation

Address reprint requests to Prof. Yury O. Tsybin, Ecole Polytechnique Fédérale de Lausanne, Biomolecular Mass Spectrometry Laboratory, BCH 4307, 1015 Lausanne, Switzerland. E-mail: yury.tsybin@epfl.ch attributed to radical intermediates ejection from the ICR trap immediately upon formation [20,21]. An alternative approach to DR-ECD is to compare ECD fragmentation patterns obtained at room-temperature $(300 \mathrm{~K})$ and cold (86 K) ICR ion trap conditions [22]. In cold ICR trap long-lived radical intermediates remain inside of the trap but do not have sufficient internal energy to initiate product ion separation and thus do not contribute to the product ion mass spectrum [9, 22]. In general, long-lived radical intermediates exhibit a higher yield of radical $\mathrm{N}$-terminal product ions, $c \cdot$ ions, and even-electron or prime C-terminal product ions, $z^{\prime}$ ions than that of short-lived intermediates, presumably as the result of increased probability of hydrogen atom transfer between ECD products $[9,23]$. Ion internal energy variation in activated ion (AI)-ECD [24] was shown to influence hydrogen atom rearrangement between ECD products and determine the ratio of radical to prime product ions $[9,21]$. Consideration of hydrogen atom loss/gain is important for correct product ion assignment and error-free peptide sequencing in proteomics [23, 25].

Implementation of electron-transfer dissociation (ETD) in ion trap mass spectrometry further catalyzed application of electron-induced fragmentation reactions in peptide and protein sequencing and post-translational modification characterization [26-30]. Compared with ECD
(C) 2009 Published by Elsevier Inc. on behalf of American Society for Mass Spectrometry. $1044-0305 / 09 / \$ 32.00$

doi:10.1016/j.jasms.2008.11.016
Published online November 27, 2008 Received May 7, 2008

Revised October 24, 2008 Accepted November 20, 2008 
FT-ICR MS of doubly charged peptides, ETD in ion trap MS typically demonstrates more abundant chargereduced radical intermediates and less extensive fragmentation pattern, indicating lower ion internal energy in ion trap-based ETD than that during ECD in an ICR ion trap [31]. Additional ion activation, or collision-induced dissociation of charge-reduced species (CRCID) [32] enhances PIA in ETD to a substantially higher degree than ion activation in ECD, especially for doubly charged precursor ions. The fragmentation pattern of ETD CRCID performed in ion trap MS seems to correlate with ECD in FT-ICR MS, whereas ETD without CRCID correlates with ECD in a low-vacuum quadrupole ion trap [33]. Indeed, it is believed nowadays that ECD and ETD produce similar fragmentation patterns. Are ECD and ETD truly similar? What method in low-vacuum ETD can be alternative or complementary to the high-vacuum ECD-based methods of peptide and protein structure analysis, such as DRECD?

Here, we first present an ETD-based method of distinguishing radical intermediates by their lifetimes as a complement to double-resonance ECD. In the following, we demonstrate the distinct differences in radical/prime PIA ratio between ECD and ETD. We rationalize the observed dependencies as a function of ion internal energy.

\section{Experimental}

\section{Sample Preparation}

Standard peptides were purchased from SigmaAldrich (Buchs, Switzerland). Peptides LLLLALLLK$\mathrm{OH}$, SDREYPLLIR-OH, and a series of $\mathrm{H}-$ RAAAAXAAAAK - OH peptides - where $X$ is one of 20 natural amino acids or a phosphorylated T, Y, or S-were produced by solid-state Fmoc chemistry on an Applied Biosystems 433A synthesizer with further purification by liquid chromatography (Protein and Peptide Synthesis Facility, Biochemistry Department, University of Lausanne, Switzerland). Peptides were dissolved in water to approximately $1 \mathrm{mM}$ concentration and further diluted in a standard spraying solution $\left(\mathrm{H}_{2} \mathrm{O} / \mathrm{CH}_{3} \mathrm{OH}\right.$ 50:50 volume ratio with $\left.1 \% \mathrm{HCOOH}\right)$ to a final peptide concentration of about $1 \mu \mathrm{M}$.

\section{ETD-based Tandem Mass Spectrometry}

ETD/CRCID experiments were performed on an ion trap mass spectrometer (HCTultra PTM discovery system, Bruker Daltonics GmbH, Bremen, Germany) by independent and subsequent application of ETD, CID, or CRCID inside the spherical ion trap [34]. Peptides were electrosprayed using a microfluidic chip-based interface (Cube source, Agilent Technologies, Cheshire, $\mathrm{UK}$ ) at a flow rate of about $300 \mathrm{~nL} / \mathrm{min}$. $\mathrm{MS}^{n}$ experiments were performed using either a manual, cutoffbased pseudoisolation of the parent ion, or a standard isolation procedure. Pseudoisolation was performed by applying a radiofrequency (RF) voltage to the ring electrode of the spherical ion trap for $40 \mathrm{~ms}$ to force all ions below a certain $\mathrm{m} / \mathrm{z}$ value (i.e., cutoff or threshold value) to be ejected out of the ion trap. CRCID was done after a manual pseudoisolation of the selected parent ion subsequently to ETD on the doubly charged peptides. ETD reagent anions from fluoranthene were generated in a chemical ionization source (reagent gas: $\mathrm{CH}_{4}$ ) and injected into the ion trap to react with the stored ionized peptides under defined conditions: 100to $120-\mathrm{ms}$ reaction time for electron transfer, active ion counting mode (ICC), and no additional delay between ETD and CRCID events. The principal difference between typically used automated CRCID (also known as supplemental activation, smart decomposition, or postactivation; subsequently referred to as autoCRCID) and the manual CRCID performed here is characterized by the ability to vary the amplitude of the activation voltage pulse and to isolate singly charged radical species before ion activation, in contrast to multiply charged radical species isolation already used in proteomics [35]. The mass spectra were acquired with a 1-min acquisition period and represent averaging over close to 100 scans; data analysis was carried out using Bruker's Data Analysis (version 3.4) software (Bruker Daltonics).

\section{ECD-based Tandem Mass Spectrometry}

ECD and AI-ECD experiments were performed on a hybrid linear ion trap LTQ FT-ICR MS (ThermoFischer Scientific, Bremen, Germany) with a 12 T superconducting magnet (Oxford Instruments Nanoscience, Abingdon, UK). Peptides were subject to electrospray ionization (ESI) with a TriVersa robot using a standard microfluidics ESI chip ( $5 \mu \mathrm{m}$ i.d. nozzles) from Advion (Ithaca, NY, USA) at a flow rate of about $200 \mathrm{~nL} / \mathrm{min}$. Desired charge states of peptide cations were isolated in LTQ (isolation window of $4 \mathrm{~m} / \mathrm{z}$ ) and transferred to the ICR ion trap for subsequent MS/MS following standard procedures [36]. ECD was performed with low-energy electrons for about $70 \mathrm{~ms}$. A $\mathrm{CO}_{2}$ IR laser was used for AI-ECD at a low power level $(<10 \mathrm{~W})$ and for short periods of time ( $<100 \mathrm{~ms})$. To account for ion magnetron motion [37], variable delay before electron injection was optimized for AI-ECD conditions (taking into account the IR laser irradiation period) and kept constant for ECD without ion activation (no IR laser power). The extent of ion activation was monitored using the radical/ prime ratio of $c_{5}$ ions of Substance $P$ as a reference [9]. Data analysis was carried out using XCalibur software (ThermoFischer Scientific).

\section{Results and Discussion}

\section{Decoupling ETD and CRCID for Peptide Analysis}

Application of various sequences of ETD-based MS/MS to neuropeptide Substance P (RPKPQQFFGLM- $\mathrm{NH}_{2}$ ) 
demonstrates distinct differences in fragmentation patterns (Figure 1). ETD directly followed by autoCRCID of singly charged species of charge-reduced radical ions generates a typical ECD FT-ICR MS type mass spectrum of Substance $\mathrm{P}$ with $z_{9}$ and most $c$ ions present $\left(c_{4}-c_{10}\right)$ (Figure 1, top) [19, 37]. As expected [32], ETD without CRCID produced an incomplete set of $c$ ions ranging from $c_{6}$ to $c_{10}$ (Figure 1, middle). In contrast to ETD data, CRCID performed on isolated charge-reduced species produced a series of $c$ ions ranging from $c_{2}$ to $c_{7}$ and a $z_{9}$ ion (Figure 1, bottom). The amplitude of additional ion activation in manual CRCID was adjusted to produce $c_{5}$ PIA comparable with ETD autoCRCID. Therefore, ETD followed by CRCID may be approximated as an addition of separately performed ETD and CRCID.
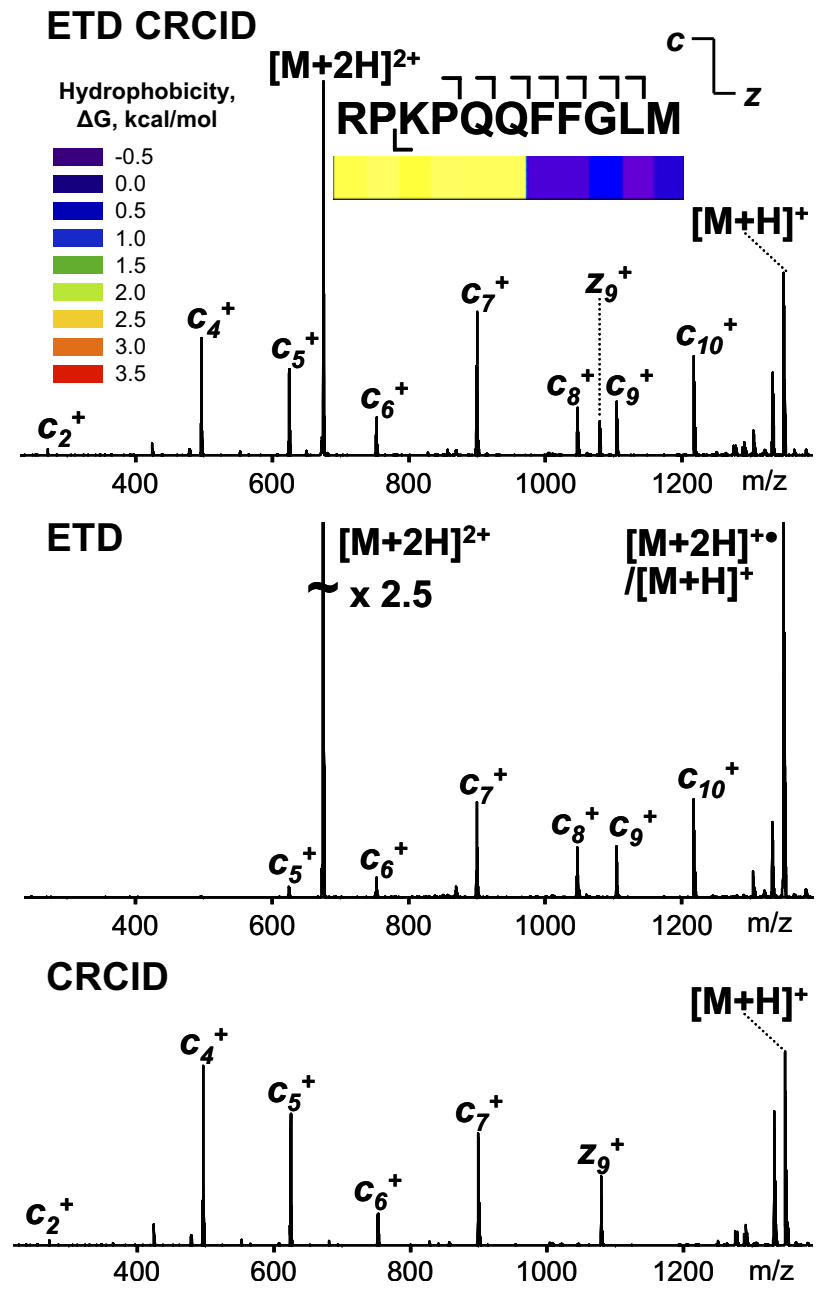

Figure 1. Tandem mass spectra of Substance $\mathrm{P}$ (RPKPQQFFGLM - $\mathrm{NH}_{2}$ ) obtained by: ETD autoCRCID (top), ETD (middle), and manual CRCID at $0.8 \mathrm{~V}_{\mathrm{p}-\mathrm{p}}$ (bottom). Strong complementarity in terms of product ion intensities as well as sequencespecific cleavages is observed. Product ions from peptide's hydrophobic (C-terminal part) region (Hessa hydrophobicity scale; [41], inset) are primarily observed in ETD mass spectra, whereas CRCID generates product ions from peptide's hydrophilic (N-terminal part) region.
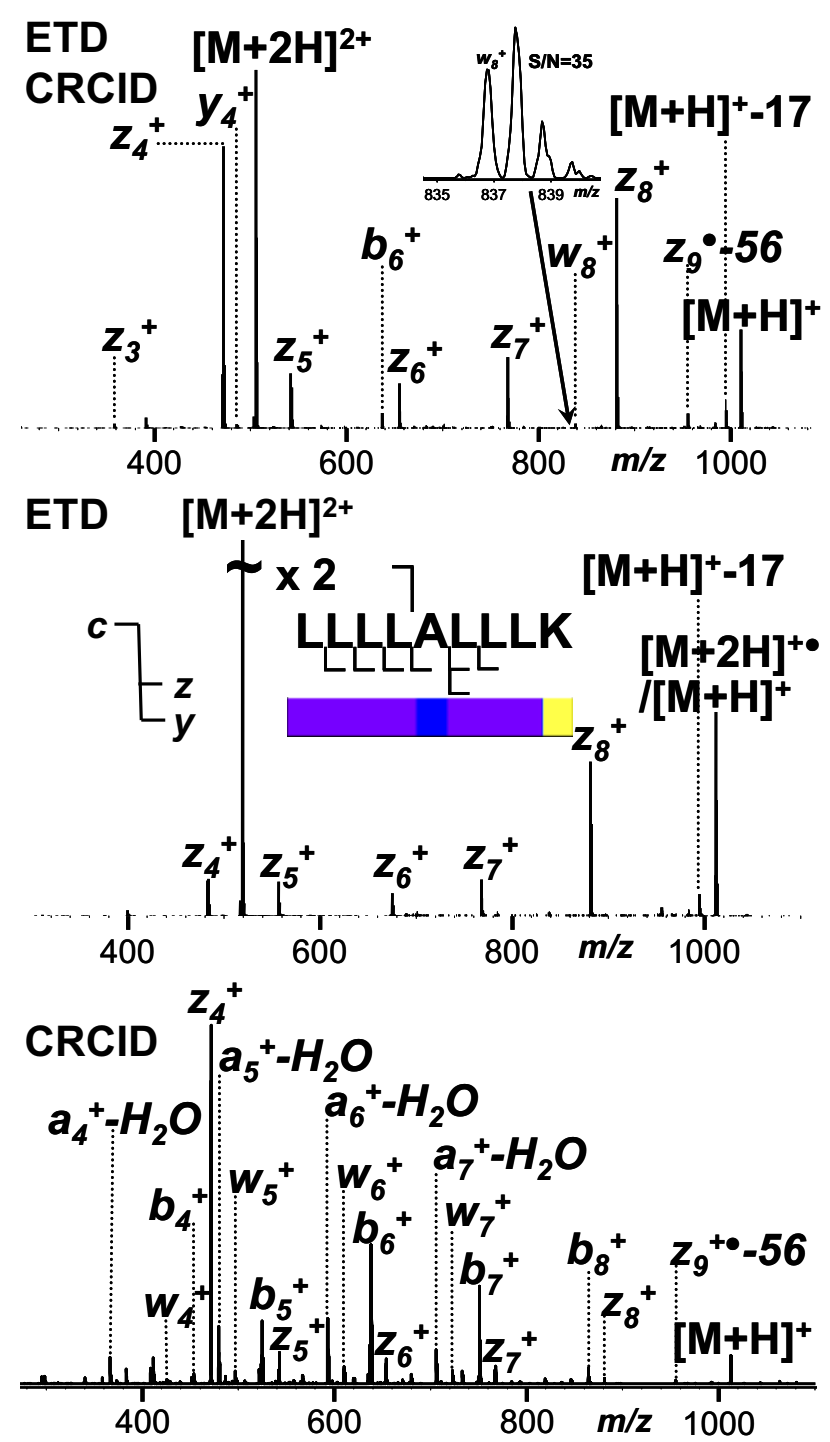

Figure 2. Tandem mass spectra of LLLLALLLK-OH obtained by: ETD autoCRCID (top), ETD (middle), and manual CRCID at $0.8 \mathrm{~V}_{\mathrm{p}-\mathrm{p}}$ (bottom). Compared with Figure 1, PIA distributions show less sequence-specific cleavages and lower degree of complementarity, presumably attributable to the location of the second charge near the C-terminus and absence of widely separated hydrophobic and hydrophilic sequence regions. CRCID produces higher than ETD yield of secondary, $w$-type, product ions.

Furthermore, we applied decoupled ETD and CRCID to a number of peptides, including synthetic peptides LLLLALLLK - OH (shown in Figure 2), SDREYPLLIR$\mathrm{OH}$, and a series of RAAAAXAAAAK $-\mathrm{OH}$ peptides (data not shown). Peptide LLLLALLLK-OH is known to produce extensive secondary fragmentation ( $w$-ion formation) under typical ECD conditions (low-energy, $\sim 1$ $\mathrm{eV}$, electrons) [38]. The autoCRCID after ETD of LLLLALLLK $-\mathrm{OH}$ results in formation of primarily ztype ions with low abundance $w_{8}$ ions and $b_{6}$ ions (Figure 2, top). Decoupling of ETD and CRCID events leads to formation of only $z$-type ions with no trace for $b$ or $w$ ions in ETD only, whereas abundant $b$ ions and pronounced $w$ ions $\left(w_{4}-w_{7}\right)$ are formed in CRCID mode at an average activation amplitude level. 


\section{Collision-Induced Activation of Radical c-Ions}

Efficiency of subsequent fragmentation of isolated ECD product ions $\left(\mathrm{MS}^{3}\right.$ in FT-ICR MS) is typically low because of a low abundance of initial product ion population and further perturbation of a product ion cloud in the ICR trap by ion isolation procedures [39]. Experimental conditions in ion trap mass analyzers allow for higher-efficiency $\mathrm{MS}^{3}$ analysis even with ETD as a first step (Figure 3) [40]. Lysine side-chain loss ( $\sim 72$ $\mathrm{Da}$ ) is the second most abundant fragmentation channel in CID of isolated $\mathrm{N}$-terminal $c_{4}$ ion (both radical and prime components are present) formed by ETD autoCRCID (Figure 3, top). CID of selected after CRCID (multiple-frequency sweep isolation: isolation center at $\mathrm{m} / \mathrm{z} 494.4$, isolation width $2.5 \mathrm{~m} / \mathrm{z}$ ) radical $c_{4}$ ion component of Substance P demonstrates a high abundance for a radical $c_{4}-72$ ion and leads to a complete depletion of parent, radical $c_{4}$ ions (Figure 3, bottom). Correspondingly, CID of a selected, mainly prime $c_{4}$-ion component shows a lower abundance of even-electron $c_{4}-72$ ions (Figure 3, middle). The inset in Figure 3 (right) shows

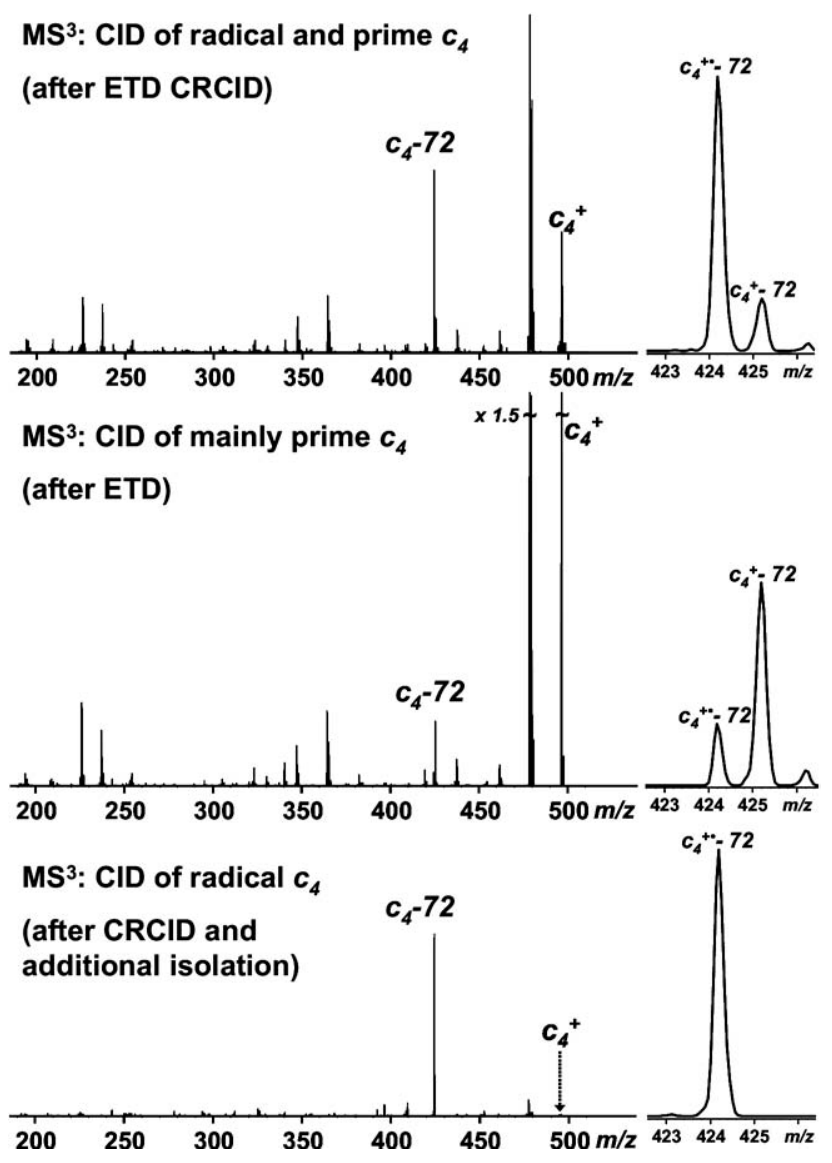

Figure 3. Dissociation of radical $c$ ions in ion trap MS: CID of $c_{4}$ ion of Substance P applied after ETD CRCID without radical isolation (top) and (middle), as well as with additional multiply frequency sweeps isolation of radical $c_{4}$ ions (bottom). The inset (right column) shows isotopically resolved radical/prime components of $c_{4} 72$ product ions (Lys side-chain loss). Note complete depletion of radical precursor ion $\left(c_{4} \cdot\right)$ upon CID (bottom). isotopically resolved distribution of radical and prime ions in the $c_{4}-72$ ion cluster to demonstrate that fragmentation pattern of CID performed on ETD CRCID products (combined radical and prime components) may be approximated by patterns of CID subsequently performed on prime and radical ETD CRCID product ions. Interestingly, CID on isolated $\mathrm{N}$-terminal $c_{7}$ ions from ETD autoCRCID of Substance P produced mainly side-chain losses with no pronounced product-ion formation, demonstrating high stability of the precursor $c_{7}$ ions, containing both radical and prime components (data not shown). Previously, CID of only even-electron $c$-type ions and radical $z$-type ions was reported [40].

\section{CRCID Amplitude Influence on Fragmentation Pattern}

PIA distributions as a function of CRCID amplitude for the peptides used exhibit similar general behavior of a sigmoidal type (Figure 4). The CRCID amplitude indicated in Figure 4 corresponds to the average activation voltage amplitude in peak-to-peak voltage $\left(\mathrm{V}_{\mathrm{p}-\mathrm{p}}\right)$, whereas applied activation voltage was varied in the range $80-130 \%$ of the indicated value according to the logic of autoCRCID ("smart decomposition") technology, in contrast to variation between 50 and $150 \%$ in a typical CID experiment. CRCID amplitude $0.0-0.4 \mathrm{~V}_{\mathrm{p}-\mathrm{p}}$ does not lead to formation of product ions, whereas product ion abundance shows saturation for CRCID amplitude $>0.7 \mathrm{~V}_{\mathrm{p}-\mathrm{p}}$. Therefore, the CRCID amplitude range $0.4-0.7 \mathrm{~V}_{\mathrm{p}-\mathrm{p}}$ shows the most pronounced impact on product-ion formation and exhibits a threshold-like behavior at around $0.55 \mathrm{~V}_{\mathrm{p}-\mathrm{p}}$. For the peptides studied in the current work we observed that the threshold dissociation energy of charge-reduced species does not substantially depend on a peptide sequence as a whole, as indicated by similar threshold values for CRCID amplitude for Substance $\mathrm{P}$ and LLLLALLLK-OH. However, differences in the local amino acid environment, e.g., bonding energy of the intermediate undissociated $\left[c^{\prime}+z \cdot\right]$ complex (interaction between neighboring amino acids or a specific conformation), produce a slight variation in threshold values for specific production formation (Figure 4, bottom inset). As presented here, data for ETD CRCID in the spherical ion trap correlate with the previously reported behavior of product ion formation upon ETD CRCID in a linear ion trap mass spectrometer (excitation voltage threshold value of $\sim 1 \mathrm{~V}$ ) [32].

\section{Complementarity of ETD/CRCID to DR ECD}

ETD data presented here can be rationalized similarly to DR ECD and cold Penning trap ECD data analysis $[20,21]$. Briefly, the change in PIA as a function of ion internal energy is believed to be peptide conformation dependent. Therefore, PIA distribution in ETD, with and without CRCID, can be considered as a measure of 

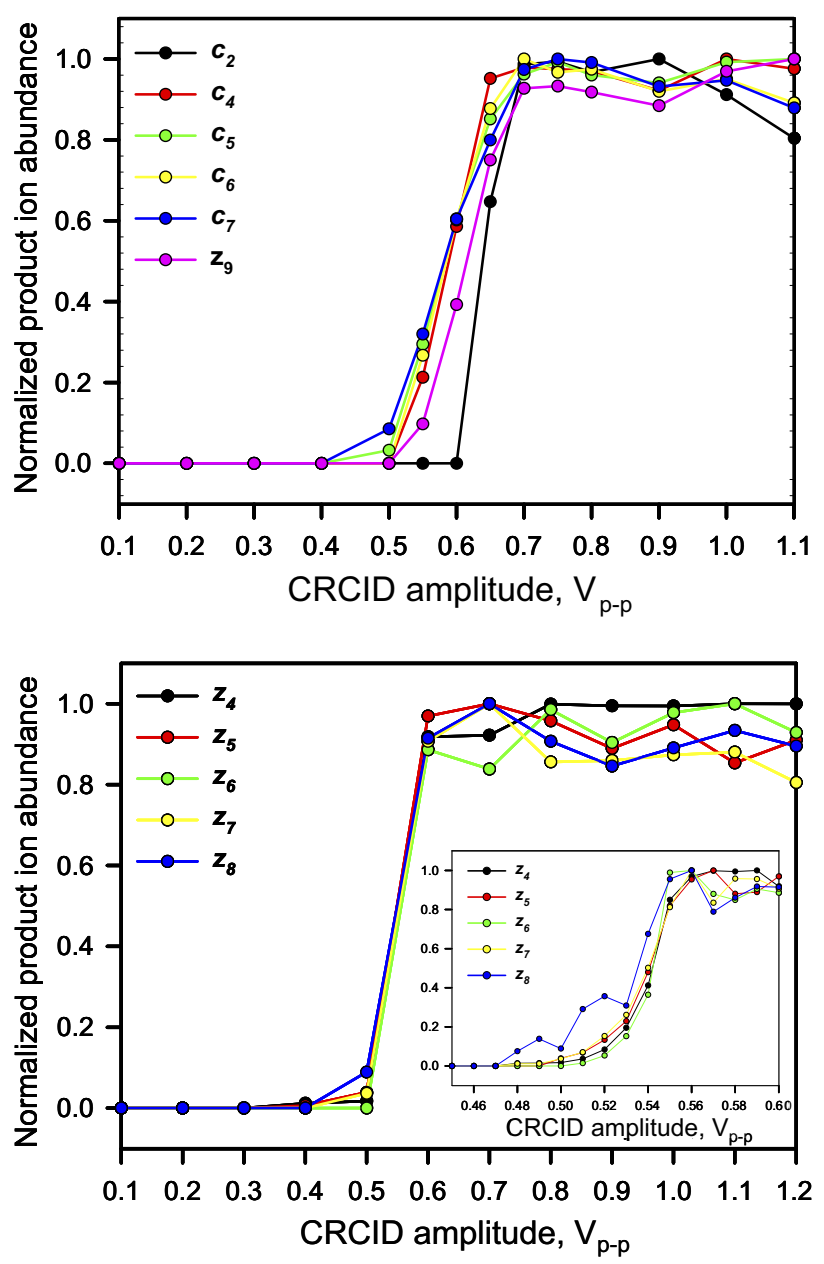

Figure 4. Product ion relative abundance as a function of CRCID activation amplitude for Substance P (RPKPQQFFGLM) (top) and LLLLALLLK (bottom) in ETD followed by manual CRCID. Expanded CRCID amplitude region $\left(0.45-0.60 \mathrm{~V}_{\mathrm{p} \text {-p }}\right)$ is shown in the inset for ETD CRCID of LLLLALLLK (bottom).

a radical intermediate, or $\left[c^{\prime}+z \cdot\right]$ complex, lifetime to reveal peptide primary and secondary (hydrogen bonding network) structural features. Indeed, both radical and prime product ions in decoupled ETD and CRCID demonstrate distinct differences. ETD and CRCID results in Figures 1 and 2 show a drastic product ion amplitude change between the different regimes of ion activation/dissociation, particularly for Substance P. We include amino acid hydrophobicity distribution along peptide sequence (Figures 1 and 2), to indicate a possible correlation between intermediate lifetime and amino acid properties [41]. Hydrophobic amino acids are characterized by a lower probability of hydrogen bond formation and may allow facile separation of products after backbone cleavage. Hydrophilic amino acids induce more hydrogen bonds that can presumably contribute to radical intermediate complex stability and require more energy for product separation. The role of the nature of amino acids, such as hydrophobicity, is to be established and requires a focused study.
Previously, ECD-based structure analysis of Substance $\mathrm{P}$ suggested dependence of PIA on peptide conformation in the gas phase: ECD in the cold ICR ion trap resulted in formation of only two ions: $c_{7}$ and $c_{10}$; DR ECD suggests an increase in intensity for ions with short lifetimes $\left(c_{8}, c_{9}\right.$, and $c_{10}$ ions) compared with that of other $c$ ions and a decrease of $c_{4}, c_{5}$, and $z_{9}$ ion abundance upon ejection of charge-reduced radical ions [21]. The reported product-ion abundance variation in DR ECD of Substance P, however, was substantial only for $c_{4}$ and $z_{9}$ ions [21, 42]. In DR ECD we constantly eject radical intermediates with long lifetimes and only short-lifetime intermediates have enough energy for reaction completion and formation of observable product ions [20]. Therefore, performing DR in ECD can be considered similar to transition from ETD with CRCID to ETD conditions: ETD proceeds at low ion internal energy and leads to formation of ions from short-lived intermediates, whereas energy addition during CRCID results in product-ion ejection from radical intermediates with longer lifetimes. Comparison of ETD and CRCID data with DR ECD and ECD in cold trap indicates higher general efficiency of the ETD method (e.g., larger amplitude of PIA variation between two regimes). That is mainly because formation of chargereduced radical intermediates in ETD is about an order of magnitude more efficient than that in ECD, which can be rationalized by lower ion internal energy in ETD versus ECD: first, precursor ions are colder in RF ion trap conditions than in the ultra high vacuum of the ICR ion trap; second, different amounts of internal energy are absorbed by the ion and available for fragmentation upon electron capture versus transfer processes [43]. Therefore, ETD with CRCID allows probing shorter lifetimes of radical intermediate complexes than DR $\mathrm{ECD}$, providing complementarity on the ion internal energy scale. The quantitative measure of the lifetime of radical complexes in ETD with CRCID can be based on PIA dependence on CRCID amplitude (Figure 4), but is yet to be established. Finally, colder precursor ions in ETD than in ECD may exhibit a different set of conformations and thus lead to the differences in behavior between DR ECD and ETD with and without CRCID.

\section{Radical Ion Formation Differs between ETD and ECD}

Decoupled ETD and CRCID of Substance P clearly separate most of $c$-type product ions, whereas $c_{7}$ ions are abundant in both regimes (Figure 1). To understand the presence of $c_{7}$ ions in both ETD and CRCID we monitored formation of radical and prime (evenelectron) $c_{7}$ ions at isotopic resolution (Figure 5, first column). Surprisingly, isotopically resolved radical and prime product ion clusters for $c_{7}$ ions demonstrate formation of only prime $c$ ions in ECD and mainly radical $c$ ions in decoupled CRCID. Both ETD and ETD autoCRCID exhibit an intermediate behavior (mixture 

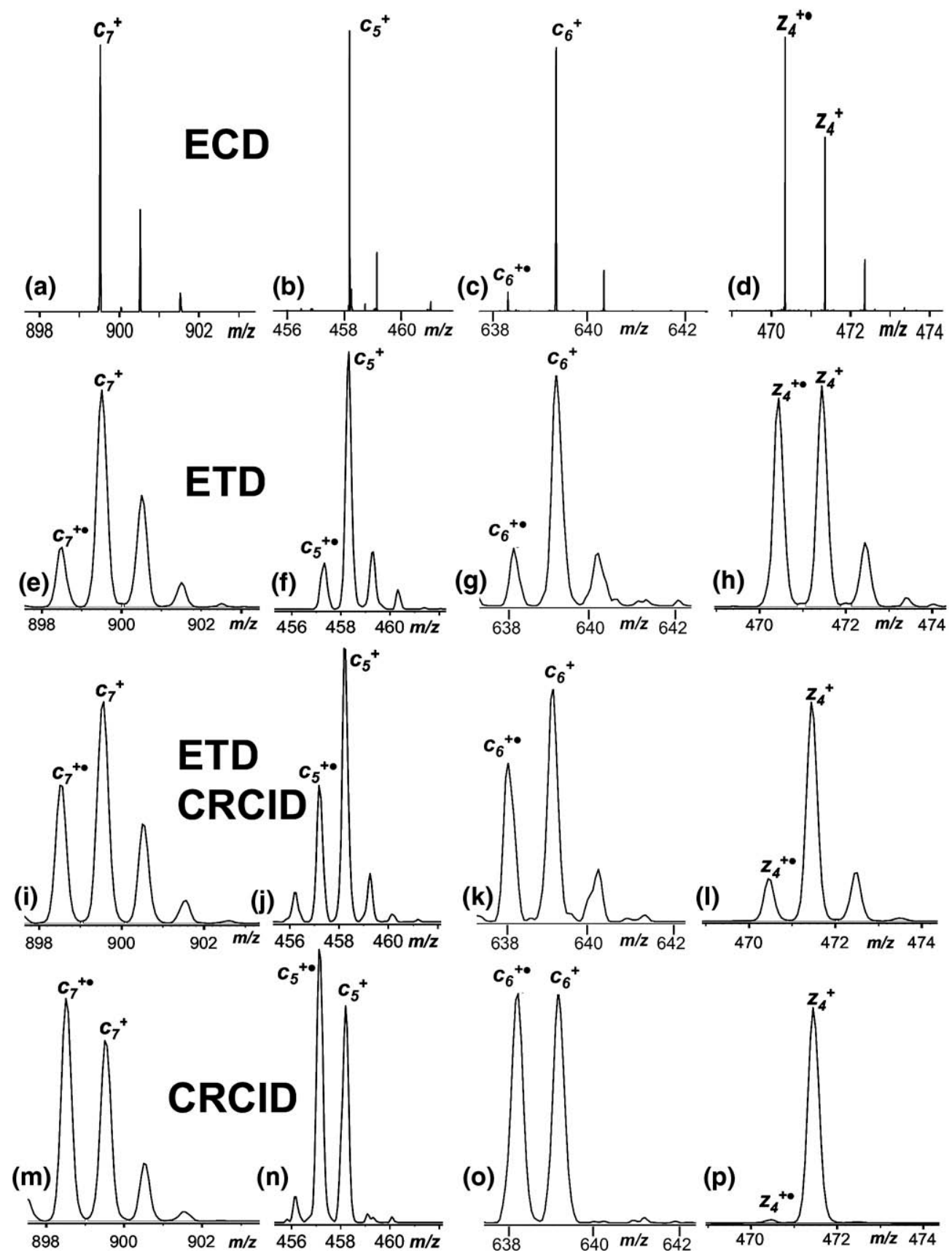

Figure 5. Radical and prime ion formation in ECD (a-d) versus ETD (e-h), ETD autoCRCID (i-1), and manual CRCID performed at $0.8 \mathrm{~V}_{\mathrm{p}-\mathrm{p}}(\mathbf{m}-\mathbf{p})$. ECD of (a) Substance $\mathrm{P},(\mathbf{b})$ RAAAAIAAAAK-OH, and (c) RAAAApTAAAAK- $\mathrm{OH}$ demonstrates formation of mainly prime (even-electron) $c_{7}, c_{5}$ and $c_{6}$ ions, correspondingly, whereas ETD with and without CRCID demonstrates a substantially higher yield of radical $c_{7}, c_{5}$, and $c_{6}$ ions $(\mathbf{e}-\mathbf{g}, \mathbf{i}-\mathbf{k})$. Similarly, ECD results in the formation of purely radical (d) $z_{4}$ ions of a peptide LLLLALLLK-OH, whereas ETD with and without CRCID demonstrates higher yield of even-electron $z_{4}$ ions $(\mathbf{h}, \mathbf{l})$. CRCID alone leads to formation of primarily radical $(\mathbf{m}) c_{7}$, (n) $c_{5}$, and (o) $c_{6}$ ions, and even-electron (p) $z_{4}$ ions.

of radical and prime components). Formation of abundant radical $c$-type ions in CRCID implies that complementary C-terminal product, if charged (z-type ion), should not have an abundant radical component in CRCID. Indeed, only radical $z$ ions at position 4 in peptide LLLLALLLK - OH upon ECD and only prime $z$ ions as a result of CRCID reaction are observed (Figure 5, right column). The general character of observations for both $c$ and z-type ions has been confirmed for a number of peptides. For instance, a pronounced difference between ECD and CRCID was observed for $c_{5}$ ions of peptide RAAAAIAAAAK among other amino acids in this peptide array (Figure 5, second column). The presence of a phospho-group induces a similar difference between the 
two fragmentation methods, as demonstrated for $c_{6}$ ions of the phosphopeptide RAAAApTAAAAK (Figure 5, third column). To our knowledge, purely radical $c$-type ions and purely prime (even-electron) z-type ions have not been observed previously in ECD FT-ICR MS of peptides and proteins. Apparently, not only for total PIA but also for radical/prime distribution, ETD CRCID is an integration of two reactions: ETD and CRCID.

Formation of abundant radical $c$ ions upon CRCID in ion trap MS, and not upon ECD in FT-ICR MS, can be rationalized by low ion internal energy-enhanced hydrogen atom rearrangement between ETD products or by the difference in conformation folding of a lower ion internal energy peptide in ion trap mass spectrometry conditions. In Substance P, for example, the peptide's $\mathrm{N}$-terminus can be bound to amino acid side chains in its C-terminal part (e.g., strong cation- $\pi$ interactions involving protonated Lys residue located close to the N-terminus and aromatic rings of $\mathrm{Phe}_{7}$ and $\mathrm{Phe}_{8}$ ), as previously suggested [22]. However, the probability of a complete hydrogen atom rearrangement to form a $c_{7}$ radical ion from a $c_{7}$ prime ion may be outweighed by an alternative mechanism of $\mathrm{N}-\mathrm{C}_{a}$ bond cleavage directly leading to formation of radical $\mathrm{N}$-terminal and prime C-terminal ions. Although ECD experimental data do not support the latter mechanism, different experimental conditions in ETD may reveal the occurrence of this reaction pathway under lower ion internal energy conditions, corresponding to Pathway II in Scheme 1 in Savitski et al. [23]. Formation of abundant prime z-type ions in CRCID can be rationalized in a similar manner. Resonant electron-transfer processes in ETD arising from specific side-chain interactions with reagent anions may also be responsible for the observed dependencies. Further improvement of our understanding of the ECD/ETD mechanism is needed to address this issue. The influence of amino acid $X$ substitution in RAAAAXAAAAK - OH on the ECD/ ETD fragmentation pattern as a function of the amino acid nature accompanied by quantum chemistry calculations may provide new insights.

Extended over Substance P and RAAAApTAAAAK$\mathrm{OH}$ sequences, the variation of $c$-type radical/ prime PIA ratio in ETD versus ECD demonstrates distinct discrepancies (Figure 6, top). For Substance P in its naturally occurring amide form, ETD versus CRCID and versus ETD autoCRCID produce very similar dependence of the radical/prime ratio for $c$
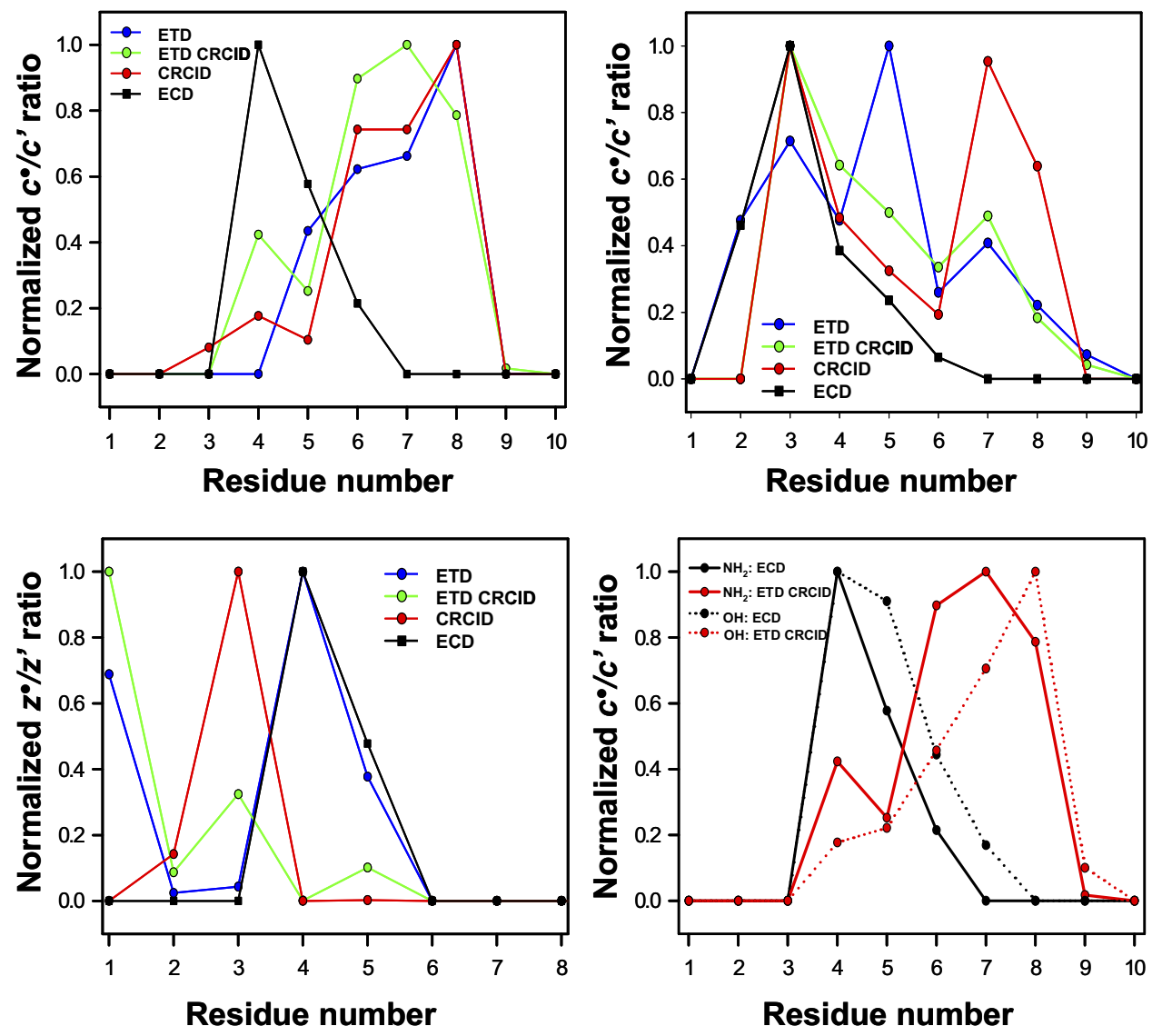

Figure 6. Product ion radical/prime ratio versus peptide sequence obtained in ECD, ETD, ETD autoCRCID, and CRCID for $c \cdot / c^{\prime}$ ratio of (top left) Substance P (RPKPQQFFGLM- $\mathrm{NH}_{2}$ ) and (top right) $\mathrm{H}$-RAAAAp'TAAAAK-OH; (bottom right) $c \cdot / c^{\prime}$ ratio comparison between Substance $\mathrm{P}$ amide form (RPKPQQFFGLM- $\mathrm{NH}_{2}$ ) and Substance $\mathrm{P}$ free acid (RPKPQQFFGLM-OH); and (bottom left) $z \cdot / z^{\prime}$ ratio of LLLLALLLK-OH. 
ions as a function of amino acid residue number (Figure 6, top left). Similar behavior is observed for RAAAApTAAAAK-OH peptide with only the $c_{5}$ ion demonstrating a sharp increase in radical/prime ratio for ETD only and not for ETD autoCRCID or CRCID only (Figure 6, top right). However, compared with ECD, the radical/prime product-ion distribution in ETD reactions is very different. The radical/prime ratios for $c_{7}-c_{10}$ ions in ECD of both Substance $\mathrm{P}$ and RAAAApTAAAAK- $-\mathrm{OH}$ are close to zero, whereas $c_{7}$ and $c_{8}$ product ions in ETD of Substance $\mathrm{P}$ and of RAAAApTAAAAK-OH demonstrate mainly radical ion contribution. When the radical/prime ratio is not negligible in ECD (Substance $\mathrm{P}$ product ions $c_{4}-c_{6}$ and RAAAApTAAAAK $-\mathrm{OH} c_{3}-c_{5}$ product ions), the radi$\mathrm{cal} /$ prime ratio in ETD, and especially in ETD CRCID, is also not equal to 0 . Dependence of the radical/prime PIA ratio as a function of amino acid residue number varies between ECD of Substance $\mathrm{P}-\mathrm{NH}_{2}$ and ECD of Substance $\mathrm{P}-\mathrm{OH}$ form (Figure 6, bottom right). ETD of both amide and free acid forms of Substance P demonstrate an anticorrelation with the ECD fragmentation pattern when the radical/prime ratio of $c$ ions is considered. Following the ECD model based on consideration of hydrogen atom rearrangement between ECD products in undissociated radical $\left[c^{\prime}+z \cdot\right]$ complex present after $\mathrm{N}-\mathrm{C}_{a}$ bond cleavage, the observed dependence may be rationalized through the conformation change between free acid and amide forms of Substance $\mathrm{P}$. The radical/prime product ion ratio dependence for $z$ ions confirms the general character of discrepancies between ECD and ETD product ion formation (Figure 6, bottom left). Clearly, for peptide LLLLALLLK-OH, the abundant radical $z_{6}$ ion at residue 3 is a result of CRCID reaction, and not ETD or ECD. Although radi$\mathrm{cal} /$ prime ratio distributions obtained for $z$ ions in ECD and ETD are almost identical, fragmentation patterns in ETD autoCRCID and CRCID demonstrate a drastic change in radical/prime ratio for $z$ ions characterized by one amino acid shift of the maxima toward the $\mathrm{N}$-terminus compared with the ETD experiment.

\section{Conclusions}

The fragmentation pattern of a peptide upon ETD with supplemental vibrational activation of charge-reduced radical intermediates (CRCID) can be accurately represented by integration of decoupled ETD and CRCID events applied to precursor peptide dications in $\mathrm{MS}^{2}$ and products of ETD reaction in $\mathrm{MS}^{3}$ experiments. Importantly, we demonstrate distinct differences between ECD and ETD radical/prime PIA ratios for both $c$ - and z-type ions for different peptides. Interestingly, CRCID after ETD can exhibit mainly radical $c$-type and prime (even-electron) z-type ions in contrast to pure prime $c$-type and radical $z$-type ions in ECD and especially AI-ECD. Although not favored by previous results, direct formation of $c$-radical and z-prime ions in ECD/ETD is suggested by current observations and needs further consideration. Differences between ECD and ETD in radical/prime PIA are observed for many but not all peptides studied and further mechanistic investigations are required to reveal their sequencedependent rules.

Similarly to DR ECD versus regular ECD and ECD in cold versus room-temperature ICR trap, decoupling of ETD and CRCID events allows us to distinguish radical intermediates by their lifetimes. Short-lived intermediates require less energy for product ion formation and appear in regular ETD mass spectra, whereas long-lived intermediates yield product ions as a function of CRCID amplitude. The high sensitivity of ion traps and greater abundance of charge-reduced radical intermediates than those of ECD FT-ICR MS provide high method efficiency. Although currently less quantitative than DR ECD, decoupled ETD and CRCID operate on a lower ion internal energy scale, allowing us to identify radical intermediates with shorter lifetimes than DR ECD, probe a different set of peptide conformations in the gas phase, and obtain higher variation in PIA between intermediates with different lifetimes. Quantitative description of radical intermediate lifetimes by decoupled ETD and CRCID would complement the DR ECD method in peptide structure analysis.

\section{Acknowledgments}

The authors gratefully acknowledge Oleg Yu. Tsybin, Andreas Brekenfeld, and Peter B. O'Connor for discussions; Sebastian Falk for the help in ETD experiments and data analysis; and Catherine Servis for help with peptide synthesis. Ecole Polytechnique Fédérale de Lausanne (EPFL) provided financial support for this work.

\section{References}

1. Zubarev, R. Protein Primary Structure Using Orthogonal Fragmentation Techniques in Fourier Transform Mass Spectrometry. Expert Rev. Proteomics 2006, 3, 251-261.

2. Zhao, C.; Xie, B.; Chan, S. Y.; Costello, C. E.; O'Connor, P. B. Collisionally Activated Dissociation and Electron Capture Dissociation Provide Complementary Structural Information for Branched Permethylated Oligosaccharides. J. Am. Soc. Mass Spectrom. 2008, 19, 138-150.

3. Siuti, N.; Kelleher, N. L. Decoding Protein Modifications Using TopDown Mass Spectrometry. Nat. Methods 2007, 4, 817-821.

4. Zubarev, R. A. Electron Capture Dissociation and Other Ion-Electron Fragmentation Reactions. In Principles of Mass Spectrometry Applied to Biomolecules, Lifshitz, C.; Laskin, J., Eds.; John Wiley and Sons: Hoboken, NJ, 2006; pp 475-518.

5. Wysocki, V. H.; Cheng, G.; Zhang, Q.; Herrmann, K. A.; Beardsley, R. L.; Hildelbrand, A. E. Peptide Fragmentation Overview. In Principles of Mass Spectrometry Applied to Biomolecules, Lifshitz, C.; Laskin, J., Eds.; John Wiley and Sons: Hoboken, NJ, 2006; pp 279-300.

6. Wells, J. M.; McLuckey, S. A. Collision-Induced Dissociation (CID) of Peptides and Proteins. Biol. Mass Spectrom. 2005, 402, 148-185.

7. Little, D. P.; Speir, J. P.; Senko, M. W.; O'Connor, P. B.; McLafferty, F. W Infrared Multiphoton Dissociation of Large Multiply-Charged Ions for Biomolecule Sequencing. Anal. Chem. 1994, 66, 2809-2815.

8. Breuker, K.; Oh, H. B.; Horn, D. M.; Cerda, B. A.; McLafferty, F. W. Detailed Unfolding and Folding of Gaseous Ubiquitin Ions Characterized by Electron Capture Dissociation. J. Am. Chem. Soc. 2002, 124, ized by 6420 .

9. Tsybin, Y. O.; He, H.; Emmett, M. R.; Hendrickson, C. L.; Marshall, A. G. Ion Activation in Electron Capture Dissociation to Distinguish between N-terminal and C-terminal Product Ions. Anal. Chem. 2007, 79, 75967602.

10. Cournoyer, J. J.; Lin, C.; Bowman, M. J.; O'Connor, P. B. Quantitating the Relative Abundance of Isoaspartyl Residues in Deamidated Proteins by Electron Capture Dissociation. J. Am. Soc. Mass Spectrom. 2007, 18, $48-56$. 
11. Pesavento, J. J.; Mizzen, C. A.; Kelleher, N. L. Quantitative Analysis of Modified Proteins and Their Positional Isomers by Tandem Mass Spectrometry: Human Histone H4. Anal. Chem. 2006, 78, 4271-4280.

12. Adams, C. M.; Kjeldsen, F.; Zubarev, R. A.; Budnik, B. A.; Haselmann, K. F. Electron Capture Dissociation Distinguishes a Single D-Amino Acid in a Protein and Probes the Tertiary Structure. J. Am. Soc. Mass Spectrom. 2004, 15, 1087-1098.

13. Adams, C. M.; Kjeldsen, F.; Patriksson, A.; van der Spoel, D.; Graslund, A.; Papadopoulos, E.; Zubarev, R. A. Probing Solution- and Gas-Phase Structures of Trp-Cage Cations by Chiral Substitution and Spectroscopic Techniques. Int. J. Mass Spectrom. 2006, 253, 263-273.

14. Breuker, K.; Oh, H. B.; Lin, C.; Carpenter, B. K.; McLafferty, F. W. Nonergodic and Conformational Control of the Electron Capture Dissociation of Protein Cations. Proc. Natl. Acad. Sci. U. S. A. 2004, 101, 14011-14016.

15. Tsybin, Y. O.; He, H.; Ben Hamidane, H.; Emmett, M. R.; Hendrickson, C. L.; Tsybin, O. Y.; Marshall, A. G. In Proceeding of the 55th ASMS Conference on Mass Spectrometry and Allied Topics, Indianapolis, IN, June 3-7, 2007.

16. Patriksson, A.; Adams, C.; Kjeldsen, F.; Raber, J.; van der Spoel, D. Zubarev, R. A. Prediction of N-C-alpha Bond Cleavage Frequencies in Electron Capture Dissociation of Trp-Cage Dications by Force-Field Molecular Dynamics Simulations. Int. J. Mass Spectrom. 2006, 248, 124-135.

17. O'Connor, P. B.; Lin, C.; Cournoyer, J. J.; Pittman, J. L.; Belyayev, M.; Budnik, B. A. Long-lived Electron Capture Dissociation Product Ions Experience Radical Migration via Hydrogen Abstraction. J. Am. Soc. Mass Spectrom. 2006, 17, 576-585

18. Kjeldsen, F.; Savitski, M. M.; Adams, C. M.; Zubarev, R. A. Determination of the Location of Positive Charges in Gas-Phase Polypeptide Polycations by Tandem Mass Spectrometry. Int. J. Mass Spectrom. 2006, 252, 204-212.

19. Tsybin, Y. O.; Haselmann, K. F.; Emmett, M. R.; Hendrickson, C. L.; Marshall, A. G. Charge Location Directs Electron Capture Dissociation of Peptide Dications. J. Am. Soc. Mass Spectrom. 2006, 17, 1704-1711.

20. Lin, C.; Cournoyer, J. C.; O'Connor, P. B. Use of a Double Resonance Electron Capture Dissociation Experiment to Probe Fragment Intermediate Lifetimes. J. Am. Soc. Mass Spectrom. 2006, 17, 1605-1615.

21. Lin, C.; Cournoyer, J. J.; O'Connor, P. B. Probing the Gas Phase Folding Kinetics of Peptide Ions by IR Activated DR-ECD. J. Am. Soc. Mass Spectrom. 2008, 19, 780-789.

22. Mihalca, R.; Kleinnijenhuis, A. J.; McDonnell, L. A.; Heck, A. J. R.; Heeren, R. M. A. Electron Capture Dissociation at Low Temperatures Reveals Selective Dissociations. J. Am. Soc. Mass Spectrom. 2004, 15, 1869-1873.

23. Savitski, M. M.; Kjeldsen, F.; Nielsen, M. L.; Zubarev, R. A. Hydrogen Rearrangement to and from Radical $\mathrm{z}$ Fragments in Electron Capture Dissociation of Peptides. J. Am. Soc. Mass Spectrom. 2007, 18, 113-120.

24. Horn, D. M.; Ge, Y.; McLafferty, F. W. Activated Ion Electron Capture Dissociation for Mass Spectral Sequencing of Larger (42 kDa) Proteins. Anal. Chem. 2000, 72, 4778-4784.

25. Liu, J.; Liang, X. R.; McLuckey, S. A. On the Value of Knowing a z. Ion for What It Is. J. Proteome Res. 2008, 7, 130-137.

26. Syka, J. E. P.; Coon, J. J.; Schroeder, M. J.; Shabanowitz, J.; Hunt, D. F. Peptide and Protein Sequence Analysis by Electron Transfer Dissociation Mass Spectrometry. Proc. Natl. Acad. Sci. U. S. A. 2004, 101, 9528-9533.

27. Coon, J. J.; Ueberheide, B.; Syka, J. E. P.; Dryhurst, D. D.; Ausio, J.; Shabanowitz, J.; Hunt, D. F. Protein Identification Using Sequential Ion/Ion Reactions and Tandem Mass Spectrometry. Proc. Natl. Acad. Sci. U. S. A. 2005, 102, 9463-9468.
28. Mikesh, L. M.; Ueberheide, B.; Chi, A.; Coon, J. J.; Syka, J. E. P.; Shabanowitz, J.; Hunt, D. F. The Utility of ETD Mass Spectrometry in Proteomic Analysis. Biochim. Biophys. Acta Proteins Proteomics 2006, 1764, 1811-1822.

29. Kaplan, D. A.; Hartmer, R.; Speir, J. P.; Stoermer, C.; Gumerov, D. Easterling, M. L.; Brekenfeld, A.; Kim, T.; Laukien, F.; Park, M. A Electron Transfer Dissociation in the Hexapole Collision Cell of a Hybrid Quadrupole-Hexapole Fourier Transform Ion Cyclotron Resonance Mass Spectrometer. Rapid Commun. Mass Spectrom. 2008, 22, 271-278.

30. McAlister, G. C.; Phanstiel, D.; Good, D. M.; Berggren, W. T.; Coon, J. J. Implementation of Electron-Transfer Dissociation on a Hybrid Linear Ion Trap-Orbitrap Mass Spectrometer. Anal. Chem. 2007, 79, 3525-3534.

31. Good, D. M.; Wirtala, M.; McAlister, G. C.; Coon, J. J. Performance Characteristics of Electron Transfer Dissociation Mass Spectrometry. Mol. Cell. Proteomics 2007, 6, 1942-1951.

32. Swaney, D. L.; McAlister, G. C.; Wirtala, M.; Schwartz, J. C.; Syka, J. E. P.; Coon, J. J. Supplemental Activation Method for High-Efficiency Electron-Transfer Dissociation of Doubly Protonated Peptide Precursors. Anal. Chem. 2007, 79, 477-485.

33. Satake, H.; Hasegawa, H.; Hirabayashi, A.; Hashimoto, Y.; Baba, T. Fast Multiple Electron Capture Dissociation in a Linear Radio Frequency Quadrupole Ion Trap. Anal. Chem. 2007, 79, 8755-8761.

34. Hartmer, R.; Kaplan, D. A.; Gebhardt, C. A.; Ledertheil, T.; Brekenfeld, A. Multiple Ion/Ion Reactions in the 3-D Ion Trap: Selective Reagent Anion Production for ETD and PTR from a Single Compound. Int. J. Mass Spectrom. 2008, 276, 82-90.

35. Wu, S. L.; Huehmer, A. F. R.; Hao, Z. Q.; Karger, B. L. On-line LC-MS Approach Combining Collision-Induced Dissociation (CID), ElectronTransfer Dissociation (ETD), and CID of an Isolated Charge-Reduced Species for the Trace-Level Characterization of Proteins with Posttranslational Modifications. J. Proteome Res. 2007, 6, 4230-4244.

36. Syka, J. E. P.; Marto, J. A.; Bai, D. L.; Horning, S.; Senko, M. W.; Schwartz, J. C.; Ueberheide, B.; Garcia, B.; Busby, S.; Muratore, T.; Shabanowitz, J.; Hunt, D. F. Novel Linear Quadrupole Ion Trap/FT Mass Spectrometer: Performance Characterization and Use in the Comparative Analysis of Histone H3 Post-translational Modifications. I. Proteome Res. 2004, 3, 621-626.

37. Tsybin, Y. O.; Hendrickson, C. L.; Beu, S. C.; Marshall, A. G. Impact of Ion Magnetron Motion on Electron Capture Dissociation Fourier Transform Ion Cyclotron Resonance Mass Spectrometry. Int. J. Mass Spectrom. 2006, 255, 144-149.

38. Cooper, H. J.; Hudgins, R. R.; Hakansson, K.; Marshall, A. G. Secondary Fragmentation of Linear Peptides in Electron Capture Dissociation. Int J. Mass Spectrom. 2003, 228, 723-728.

39. Tsybin, Y. O.; Witt, M.; Baykut, G.; Kjeldsen, F.; Hakansson, P. Combined Infrared Multiphoton Dissociation and Electron Capture Dissociation with a Hollow Electron Beam in Fourier Transform Ion Cyclotron Resonance Mass Spectrometry. Rapid Commun. Mass Spectrom. 2003, 17, 1759-1768.

40. Han, H. L.; Xia, Y.; McLuckey, S. A. Ion Trap Collisional Activation of $\mathrm{c}$ and $\mathrm{z}$. Ions Formed via Gas-Phase Ion/Ion Electron-Transfer Dissociation. J. Proteome Res. 2007, 6, 3062-3069.

41. Hessa, T.; Kim, H.; Bihlmaier, K.; Lundin, C.; Boekel, J.; Andersson, H.; Nilsson, I.; White, S. H.; von Heijne, G. Recognition of Transmembrane Helices by the Endoplasmic Reticulum Translocon. Nature 2005, 433, 377-381.

42. Lin, C.; Cournoyer, J. J.; O'Connor, P. B. In Proceedings of the 55th ASMS Conference on Mass Spectrometry and Allied Topics, Indianapolis, IN, June $3-7,2007$.

43. Sobczyk, M.; Simons, J. The Role of Excited Rydberg States in Electron Transfer Dissociation. J. Phys. Chem. B 2006, 110, 7519-7527. 